\title{
Human papillomavirus vaccine in boys: background rates of potential adverse events
}

\section{Hazel J Clothie FIBMS, MSc, MAppEpid, Epidemiologist and Surveillance Manager, ${ }^{1}$ and Senior Lecturer and PhD Student ${ }^{2}$ \\ Katherine J Lee $\mathrm{BSc}, \mathrm{MSc}, \mathrm{PhD}$ Biostatician \\ Vijaya Sundararajan MD, MPH Associate Professor \\ Jim P Buttery FRACP, MD, MSC Director, ${ }^{1}$ and Paediatric Infectious Diseases Physician $^{5}$ \\ Nigel W Crawford BM BS, MPH, PhD, Deputy Director,' and Paediatrician and Medical Head Immunisation Services \\ 1 Surveillance of Adverse Events Following Vaccination in the Community (SAEFVIC), Murdoch Childrens Research Institute, Melbourne, VIC
Mearch Institute, \\ 2 School of Population and Global Health \\ University of Melbourne Melbourne, VIC. \\ 3 Clinical Epidemiology and Biostatistics Unit, Murdoch Childrens Research Institute, Melbourne, VIC. 4 Department of Medicine Monash University, Melbourne, VIC \\ 5 Department of Infectious Diseases and Department of Paediatrics, \\ Monash University, \\ Melbourne, VIC \\ 6 Department of General Medicine Royal Children's Hospital, Melbourne, VIC \\ hazel.clothier@ mcri.edu.au}

MJA 2013; 198: 554-558 doi: 10.5694/mjal2.1175

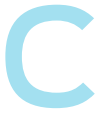

ervical cancer is the most common cancer affecting women in developing countries. It is caused by persistent infection with specific types of human papillomavirus (HPV). ${ }^{1}$ Quadrivalent human papillomavirus (4vHPV) vaccine is a recombinant vaccine administered as a threedose course to provide protection against four types of $\operatorname{HPV}(6,11,16$ and 18$).^{2}$ The vaccine is highly efficacious for the four included types, of which 16 and 18 are reported to cause $70 \%$ of cervical cancers and 6 and 11 cause anogenital warts. ${ }^{1,3} 4 \mathrm{vHPV}$ vaccination was introduced under the Australian National Immunisation Program (NIP) in April 2007 for adolescent girls, with an initial catch-up program including women up to 26 years of age. The current ongoing funded program is only for girls in the first year of high school (aged 12-13 years). Recent data suggest that the $4 \mathrm{vHPV}$ vaccination program has caused a rapid decline in genital wart presentations in females, ${ }^{4,5}$ and there are early indications of a reduction in high-grade cervical dysplasias. ${ }^{6}$

Following advice from the Australian Technical Advisory Group on Immunisation, vaccination of males was recommended as a cost-effective intervention by the Pharmaceutical Benefits Advisory Committee in November 2011. ${ }^{7}$ Accordingly, 4vHPV vaccination for boys has been added to the Australian NIP, commencing in 2013 and targeting boys aged 12-13 years in a school-based program, with a catch-up program over 2 years for boys aged $14-16$ years. ${ }^{7,8}$ The program aims to reduce the incidence of HPV disease in males, such as anogenital warts and anal intraepithelial neoplasia, ${ }^{9}$ and reduce sexual pathways of virus transmission. Australia will be the first nation to implement HPV vaccination for boys in a national program.

Vaccines, as with any medicine, have potential adverse reactions varying from mild and expected to rare and/or serious events. Vaccination may cause such events - the nature of adverse events following immuni-

\begin{abstract}
Objectives: To determine background rates of potential adverse events following immunisation (AEFI) before expansion of the quadrivalent human papillomavirus (4VHPV) vaccination program to adolescent boys.

Design, patients and setting: Retrospective analysis of hospital discharge data obtained from the Victorian Admitted Episodes Dataset and emergency department visit data obtained from the Victorian Emergency Minimum Dataset for boys aged 12 to < 16 years during the period 1 July 2004 to 30 June 2009.
\end{abstract}

Main outcome measures: Numbers of and incidence rates for Guillain-Barré syndrome, anaphylaxis, seizures, syncope and other potential AEFI from 1 July 2004 to 30 June 2009, and estimated numbers of events after 4VHPV vaccination assuming no association (other than temporal) with the vaccine.

Results: We estimated background rates of neurological and allergic events in adolescent boys to be 252.9 and 175.2 per 100000 person-years, respectively. Assuming an $80 \%$ vaccination rate with three doses per person - which equates to 1440000 doses administered nationally per year in the first 2 years of the program - about 2.4 episodes of Guillain-Barré syndrome would be expected to occur in the 6 weeks following vaccination. Within 1 day of vaccination, about 3.9 seizures, 0.3 episodes of anaphylaxis and 6.5 acute allergy presentations would be expected.

Conclusions: Routinely collected health outcome administration data can inform postlicensure safety surveillance of target conditions that might be perceived as AEFI.

sation (AEFI) and the timing of onset after vaccination are important factors when assessing causation. Adverse events may also coincide temporally with vaccine administration by chance. To interpret postlicensure surveillance data, it is useful to know the background rates of common and rare potential adverse events before introduction of the vaccine. ${ }^{10,11}$ With this understanding, increases above background rates can be rapidly identified, which can assist with the evaluation and reporting of potential vaccineassociated adverse event rates.

The mass school-based introduction of female $4 \mathrm{vHPV}$ vaccination raised a number of well publicised initial safety concerns, including "scares" regarding potential episodes of anaphylaxis and multiple sclerosis after vaccination. ${ }^{12-14}$ In addition, a mass psychogenic reaction was seen in a Melbourne school vaccination environment, ${ }^{15}$ with syncope and syncopal seizures occurring in response to the vaccination process. ${ }^{16}$ Such spurious events may arise from the psychological impact of the vaccination process, particularly when using mass vaccination strategies in a school-based teenaged population.
Release of the $4 \mathrm{vHPV}$ vaccine to boys has the advantage of adverse event information from prelicensure clinical trials and postlicensure surveillance of adverse events arising from administration to adolescent girls. However, additional information on the background rates of potential adverse events in teenaged boys is critical for assessing the safety of this vaccination program.

Our aim was to explore the use of routinely collected information for estimating potential adverse event rates. We used population-level health outcome administration data to describe the background rates of potential AEFI before the introduction of $4 \mathrm{vHPV}$ vaccination for boys into the NIP in Australia, and to estimate numbers of a range of neurological, allergic and other events that can be expected following vaccination, assuming temporal association with administration of vaccine but no other association.

\section{Methods}

Two statewide Victorian datasets were accessed - the Victorian Admitted Episodes Dataset (VAED; hospital 
discharge data) and the Victorian Emergency Minimum Dataset (VEMD; emergency department visit data) - both of which include International Classification of Diseases 10th revision Australian modification (ICD-10-AM) codes. The data included a unique identifier that enabled linking of individuals across the datasets, but were otherwise nonidentifying, according to Victorian Department of Health data linkage protocols. ${ }^{17}$ Ethics approval for the study was provided by the VAED and VEMD data custodians.

Multiple records of the same event within a dataset or across datasets for example, a person presenting at emergency who is subsequently admitted, or a person admitted to hospital who is then discharged to a different hospital or to home and who later returns with continuation of the same episode (with each presentation recorded as a separate event) - were linked via the unique identifier. All events occurring within 28 days of a previous event were combined into a single episode.

The data that we analysed comprised all episodes that occurred in boys aged 12 to $<16$ years and were recorded in the VAED and/or VEMD with one of the ICD-10-AM codes listed in Box 1 and an admission or presentation date from 1 July 2004 to 30 June 2009. ${ }^{18}$ Conditions selected for inclusion are rare adverse events, conditions that patients are likely to present to hospitals with after vaccination, and conditions that have previously been raised as potential sources of concern in Australia and overseas. $^{10,19}$

Age was taken to be the youngest age at which an episode occurred, and records were excluded from the analysis if sex was recorded inconsistently among records with the same unique identifier. Some records had more than one ICD-10-AM code, and these were preserved. Events with an interstate or overseas postcode were excluded, but those with "unknown" (8888 and 9988) and "of no fixed abode" (1000) postcodes were preserved under the assumption that these occurred in Victoria. Episodes that were ongoing from the 3 months before the study period, the washout period (31 March to 30 June 2004), were also excluded.

Events were described as the number of episodes and the number of first events. An episode was considered a discrete event if it occurred more than 28 days after a prior event in the same individual, as patients were deemed to still be "at risk" of the same event during their recovery from an acute condition. First events were defined as the first time a condition was diagnosed in each patient during the study period. First events are more relevant for chronic conditions and episodes are more relevant for acute conditions.

We calculated background annual incidence rates as the number of events during the 5-year study period divided by the population at risk during this period, using Australian Bureau of Statistics 2006 mid-year resident population data for males. ${ }^{20}$

The analysis was restricted to boys aged 12 to $<16$ years - the target age range for vaccination. We used these background rates to estimate the number of events expected within 1 day, 1 week and 6 weeks of vaccination per 100000 vaccinees. We then estimated the expected number of events for each condition 1 day, 1 week and 6 weeks after vaccination across Australia following the introduction of $4 \mathrm{vHVP}$ into the NIP, assuming there is no association (other than temporal) with the vaccine.

Seasonal variation was analysed by graphing the number of first events or episodes by month of presentation. As the numbers of chronic neurological presentations in the study group were small, they were combined and compared with numbers of all-age presentations in males for individual neurological conditions. For multiple sclerosis, data were also presented omitting presentations in the first 12 months of the study period to assess the effectiveness of the study's 3month washout period.

\section{Results}

The numbers of and incidence rates for potential AEFI in boys aged 12 to $<16$ years are shown in Box 2, and the estimated numbers of cases of potential AEFI per 100000 adolescent boys

\section{Conditions included in the study}

ICD-10-AM codes

\section{Neurological}

Guillain-Barré syndrome*

Transverse myelitis*

G61.0

Multiple sclerosis*

G37.3

Optic neuritis*

ADEM

G35

$\mathrm{H} 46, \mathrm{G} 36.0$

Bell's palsy

G04.0

Syncope

Seizures

G51.0

R55

Allergic

Anaphylaxis

R56, R56.0, R56.8

Urticaria

Serum sickness

T78.2, T88.6

Adverse effect of drug or

medication ${ }^{\dagger}$

Other

Adverse events

L50.O, L50.1, L50.9

T80.6

T88.7

T78.8, T78.9, T88.1, T78.3

-10-AM = International Classification of Diseases 10th revision Australian modification. ADEM = acute disseminated

encephalomyelitis. * Conditions considered chronic. $†$ Not otherwise specified.

that would occur, even in the absence of vaccine, are shown in Box 3. Assuming an $80 \%$ vaccination rate with three doses per person — which equates to about 480000 boys vaccinated and a total of 1440000 doses administered nationally per year in the first 2 years of the program about 2.4 episodes of Guillain-Barré syndrome would be expected to occur within 6 weeks of vaccination. In addition, about 3.9 seizures and 6.5 acute allergy presentations would be expected to occur within 1 day of vaccination, including 0.3 episodes of anaphylaxis.

There was minimal seasonal variation in the occurrence of potential AEFI (Box 4, Box 5). However, repeating this analysis with a larger number of neurological presentations (using data for all age groups) revealed a notable peak in the number of multiple sclerosis presentations in July. This peak was reduced but not eliminated when the washout period was increased to 15 months (Box 4).

\section{Discussion}

Using statewide morbidity data, we estimated background rates of neurological and allergic events in adolescent boys in Victoria to be 252.9 and 175.2 per 100000 person-years, respectively. Such adverse events 
2 Numbers of and incidence rates for potential AEFI in boys aged 12 to $<16$ years (Victoria, July 2004 - June 2009)

First events

\begin{tabular}{cc}
\hline $\begin{array}{c}\text { No. of } \\
\text { events }\end{array}$ & $\begin{array}{c}\text { Incidence rate }(95 \% \mathrm{Cl}) \text { per } \\
100000 \text { person-years }\end{array}$
\end{tabular}

\section{Neurological}

Guillain-Barré syndrome

Transverse myelitis

Multiple sclerosis

Optic neuritis

ADEM

Bell's palsy

Syncope

Seizures

Total

Allergic

Anaphylaxis

Urticaria

Serum sickness

Allergic reaction

Total

Other

Total

10
2
8
60
807
66
1516

10

2

2

4

8

60

807

666

1516

49

620

23

495

1125

7

\begin{abstract}
1.46 ( 0.56 to 2.37 )
0.29 ( -0.11 to 0.70$)$

0.29 ( -0.11 to 0.70$)$

0.59 (0.01 to 1.16)

0.17 (0.45 to 1.90$)$

8.78 (6.56 to 11.00 )

118.0 (109.9 to 126.2)

97.4 (90.0 to 104.8)

221.7 (210.6 to 232.9)
\end{abstract}

$$
\begin{gathered}
7.17 \text { (5.16 to } 9.17) \\
90.7 \text { ( } 83.6 \text { to } 97.8) \\
3.4(2.0 \text { to } 4.7) \\
72.4 \text { (66.0 to } 78.8) \\
164.6 \text { (154.9 to } 174.2)
\end{gathered}
$$

1.02 (0.27 to 1.78)

Episodes

No. of Incidence rate $(95 \% \mathrm{Cl})$ per
events 100000 person-years

$\mathrm{AEFI}=$ adverse events following immunisation. $\mathrm{ADEM}=$ acute disseminated encephalomyelitis.

may be mistakenly assumed to be caused by vaccination, owing to temporal association, when the $4 \mathrm{vHPV}$ vaccination program is expanded to include adolescent boys. ${ }^{10}$ Postlicensure safety assessments of $4 \mathrm{vHPV}$ vaccine programs in adolescent girls have shown little evidence of increased risk of neuro- logical and allergic adverse events after vaccination. 3,21,22

Expected rates of potential AEFI in recent studies vary widely, but direct comparisons are restricted because of differences in methods, health care systems and data collection and analyses. ${ }^{10,11,23}$ In particular, caution is required when using emergency pres- entation databases as these may record preliminary diagnoses, rather than final diagnoses. Studies limited to analysis of ICD-10 coded data, such as ours, lack the rigour of diagnosis verification and conformity to standardised case definitions, although coding standards are maintained. Our study identified higher reporting rates for anaphylaxis compared with similar studies. ${ }^{10,11}$ While data aberrations are possible, marked increases in anaphylaxis rates Australia and the United States over the past two decades may play a part. ${ }^{24,25}$

Background rates of potential AEFI and consequent thresholds for safety flags should not be informed merely using data on adolescent girls because sex-related differences could cause misinterpretation of potential signals. ${ }^{10,11}$ For example, the rate of adolescent boys presenting with a first multiple sclerosis event in the 6 weeks following vaccination would be expected to be one-third of the rate seen for adolescent girls assuming no relationship with vaccine other than temporal. $^{26}$

In our study, we used a 3-month washout period to attempt to remove the risk of categorising events as incident cases when they were part of a pre-existing illness than was ongoing from before the study period. How-

\begin{tabular}{|c|c|c|c|c|c|c|}
\hline & & & & & & \\
\hline & 1 day & 1 week & 6 weeks & 1 day & 1 week & 6 weeks \\
\hline \multicolumn{7}{|l|}{ Neurological } \\
\hline Guillain-Barré syndrome & $0(0.00-0.01)$ & $0.03(0.01-0.05)$ & $0.17(0.06-0.27)$ & $0(0.00-0.01)$ & $0.03(0.01-0.05)$ & $0.19(0.08-0.29)$ \\
\hline Transverse myelitis & $0(0.00-0.00)$ & 0.01 (0.00-0.01) & $0.03(0.00-0.08)$ & $0(0.00-0.00)$ & $0.01(0.00-0.02)$ & $0.05(0.00-0.11)$ \\
\hline Multiple sclerosis & $0(0.00-0.00)$ & 0.01 (0.00-0.01) & $0.03(0.00-0.08)$ & $0(0.00-0.00)$ & 0.01 (0.00-0.01) & $0.03(0.00-0.08)$ \\
\hline Optic neuritis & $0(0.00-0.00)$ & $0.01(0.00-0.02)$ & $0.07(0.00-0.13)$ & $0(0.00-0.00)$ & $0.02(0.00-0.03)$ & $0.10(0.02-0.18)$ \\
\hline ADEM & $0(0.00-0.01)$ & $0.02(0.01-0.04)$ & $0.15(0.05-0.25)$ & $0(0.00-0.01)$ & $0.03(0.01-0.05)$ & $0.19(0.08-0.29)$ \\
\hline Bell's palsy & $0.02(0.02-0.03)$ & $0.17(0.13-0.21)$ & $1.01(0.75-1.26)$ & $0.02(0.02-0.03)$ & $0.17(0.13-0.21)$ & $1.01(0.75-1.26)$ \\
\hline Syncope & $0.32(0.30-0.35)$ & $2.26(2.11-2.42)$ & 13.57 (12.64-14.51) & $0.33(0.31-0.36)$ & $2.33(2.17-2.49)$ & 13.98 (13.03-14.93) \\
\hline Seizures & $0.27(0.25-0.29)$ & $1.87(1.73-2.01)$ & 11.20 (10.35-12.05) & $0.33(0.31-0.35)$ & $2.33(2.17-2.48)$ & 13.96 (13.01-14.91) \\
\hline Total & $0.61(0.58-0.64)$ & $4.25(4.04-4.46)$ & $25.50(24.22-26.78)$ & $0.69(0.66-0.72)$ & $4.85(4.62-5.07)$ & $29.08(27.71-30.45)$ \\
\hline \multicolumn{7}{|l|}{ Allergic } \\
\hline Anaphylaxis & $0.02(0.01-0.03)$ & $0.14(0.10-0.18)$ & $0.82(0.59-1.05)$ & $0.02(0.01-0.03)$ & $0.14(0.10-0.18)$ & $0.86(0.62-1.09)$ \\
\hline Urticaria & $0.25(0.23-0.27)$ & $1.74(1.60-1.87)$ & $10.43(9.61-11.25)$ & $0.26(0.24-0.28)$ & $1.81(1.67-1.95)$ & $10.88(10.04-11.72)$ \\
\hline Serum sickness & 0.01 (0.01-0.01) & $0.06(0.04-0.09)$ & $0.39(0.23-0.54)$ & 0.01 (0.01-0.01) & $0.06(0.04-0.09)$ & $0.39(0.23-0.54)$ \\
\hline Allergic reaction & $0.20(0.18-0.22)$ & 1.39 (1.27-1.51) & 8.33 (7.59-9.06) & $0.21(0.19-0.22)$ & $1.45(1.32-1.57)$ & $8.70(7.95-9.44)$ \\
\hline Total & $0.45(0.42-0.48)$ & $3.15(2.97-3.34)$ & $18.92(17.82-20.03)$ & $0.48(0.45-0.51)$ & $3.36(3.17-3.55)$ & $20.15(19.01-21.29)$ \\
\hline \multicolumn{7}{|l|}{ Other } \\
\hline Total & $0(0.00-0.00)$ & $0.02(0.01-0.03)$ & $0.12(0.03-0.20)$ & $0(0.00-0.00)$ & $0.02(0.01-0.03)$ & $0.12(0.03-0.20)$ \\
\hline
\end{tabular}
ever, the 3-month washout period did

3 Estimated numbers of cases of potential AEFI in vaccinated boys aged 12 to $<16$ years, assuming no relationship with vaccine*

No. of first events per 100000 population

No. of episodes per 100000 population 
4 Numbers of first events of chronic conditions, by month (Victoria, July 2004 June 2009)

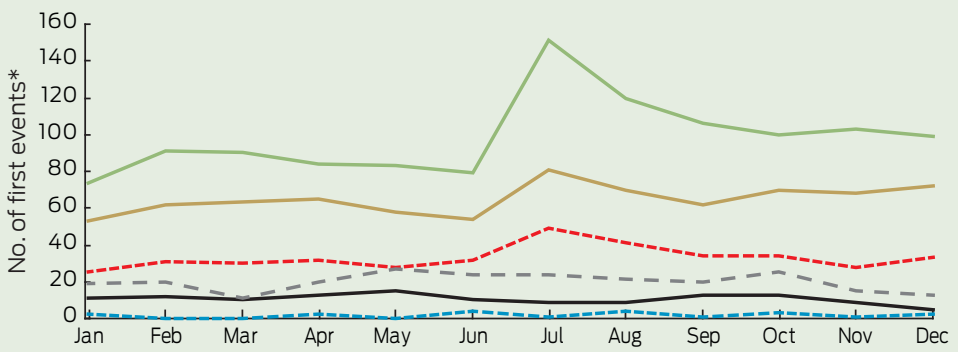

---- Chronic neurological conditions included in the analysis, boys aged 12 to $<16$ years ${ }^{\dagger}$ ---- Guillain-Barré syndrome, males of all ages

- Transverse myelitis, males of all ages

- Multiple sclerosis (3-month washout period), males of all ages

- Multiple sclerosis (15-month washout period), males of all ages

- - Optic neuritis, males of all ages

* Data are numbers of first events for chronic neurological conditions analysed in boys aged 12 to $<16$ years and numbers of presentations for individual neurological conditions in males of all ages. † Conditions included were Guillain-Barré syndrome, transverse myelitis, multiple sclerosis and optic neuritis.

\section{Number of episodes of acute conditions by month in boys aged 12 to $<16$ years} (Victoria, July 2004 - June 2009)

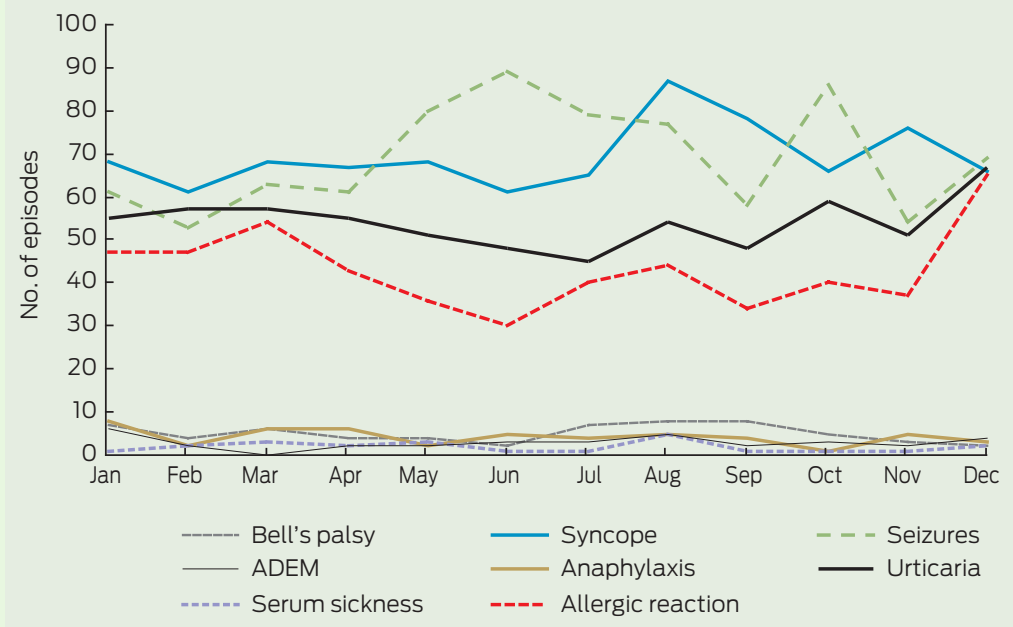

ADEM = acute disseminated encephalomyelitis.

not remove this issue for multiple sclerosis. While our study showed little seasonal variation in potential AEFI, school-based vaccination programs are conducted in blocks (as convenient to the vaccine schedule and the school year), which may give rise to false signal detection. Specific investigation of appropriate washout periods, as well as seasonal variation in the occurrence potential AEFI and implementation of the vaccine program, must therefore be explored before conducting in-depth analyses for specific conditions or extrapolating data to other jurisdictions.

In Victoria, first-dose 4vHPV vaccine coverage for adolescent girls has tive of causal association. While current passive surveillance system reporting is likely to underascertain postvaccination events, prior knowledge of expected numbers of events are valuable in helping determine whether reports or clusters of reports represent real safety flags that require urgent investigation. ${ }^{26}$

Our data highlight the value of statewide and nationwide health datasets in providing information that can improve public safety. In addition to establishing background rates of diseases, international systems such as those in Denmark and the US, have been used to link vaccination databases to health care event databases, enabling direct investigation of potential associations with adverse events. $^{29-31}$ These methods, conducted in accordance with state and federal privacy protections, offer a promising future for further improving vaccine safety in Australia. ${ }^{32}$

Routinely collected state health outcome data can enable informed postlicensure safety surveillance of conditions that may be perceived as AEFI. When the $4 \mathrm{vHPV}$ vaccine program is expanded to adolescent boys, such data can be used for targeted active surveillance of potential vaccine safety flags.

Acknowledgements: We thank Lalitha Sundaresan of the Victorian Department of Health for initial data extractions.

Competing interests: Hazel Clothier and Jim Buttery are members of the Advisory Committee on the Safety of Vaccines.

Received 29 Nov 2012, accepted 10 Mar 2013.

1 Cutts FT, Franceschi S, Goldie S, et al. Human papillomavirus and HPV vaccines: a review. Bull World Health Organ 2007; 85: 719-726.

2 McCormack PL, Joura EA. Quadrivalent human papillomavirus (types $6,11,16,18$ ) recombinant

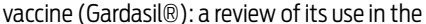
prevention of premalignant genital lesions, genital cancer and genital warts in women. Drugs 2010; 70: 2449-2474.

3 Lu B, Kumar A, Castellsagué X, Giuliano AR. Efficacy and safety of prophylactic vaccines against cervical HPV infection and diseases among women: a systematic review and metaanalysis. BMC Infect Dis 2011; 11: 13.

4 Fairley CK, Hocking JS, Gurrin LC, et al. Rapid decline in presentations of genital warts after the implementation of a national quadrivalent human papillomavirus vaccination programme for young women. Sex Transm Infect 2009; 85: 499-502.

5 Donovan B, Franklin N, Guy R, et al. Quadrivalent human papillomavirus vaccination and trends in genital warts in Australia: analysis of national sentinel surveillance data. Lancet Infect Dis 2011; 11: 39-44

6 Garland SM, Skinner SR, Brotherton JM. Adolescent and young adult HPV vaccination in 
Australia: achievements and challenges. Prev Med 2011; 53 Suppl 1: S29-S35.

7 Pharmaceutical Benefits Advisory Committee. November 2011 PBAC meeting outcomes positive recommendations. http://www. health.gov.au/internet/main/publishing.nsf/ Content/DF3D2BF61025D73CCA25796600 80CA50/\$File/PBAC\%200utcomes\%20Nov\% 202011\%20-\%20Positive\%20recommen dations.pdf (accessed Feb 2013).

8 Department of Health and Ageing. HPV vaccine extended to boys [media release]. $12 \mathrm{Jul} 2012$. http://www.health.gov.au/internet/ministers/ publishing.nsf/Content/mr-yrl2-tp-tp059.htm (accessed Feb 2013).

9 Hillman RJ, Giuliano AR, Palefsky JM, et al. Immunogenicity of the quadrivalent human papillomavirus (type 6/11/16/18) vaccine in males 16 to 26 years old. Clin Vaccine Immunol 2012; 19: 261-267.

10 Siegrist CA, Lewis EM, Eskola J, et al. Human papilloma virus immunization in adolescent and young adults: a cohort study to illustrate what events might be mistaken for adverse reactions. Pediatr Infect Dis J 2007; 26: 979-984.

1 Callréus T, Svanström H, Nielsen NM, et al. Human papillomavirus immunisation of adolescent girls and anticipated reporting of immune-mediated adverse events. Vaccine 2009; 27: 2954-2958

12 Gold MS, McIntyre P. Human papillomavirus vaccine safety in Australia: experience to date and issues for surveillance. Sex Health 2010; 7 : 320-324.

13 Chang J, Campagnolo D, Vollmer TL, Bomprezzi R. Demyelinating disease and polyvalent human papilloma virus vaccination. J Neurol Neurosurg Psychiatry 2011; 82: 1296-1298.

14 Brotherton JM, Gold MS, Kemp AS, et al. Anaphylaxis following quadrivalent human papillomavirus vaccination. CMAJ 2008; 179: 525-533.
15 Buttery JP, Madin S, Crawford NW, et al. Mass psychogenic response to human papillomavirus vaccination. Med J Aust 2008; 189: 261-262.

16 Crawford NW, Clothier HJ, Elia S, et al. Syncope and seizures following human papillomavirus vaccination: a retrospective case series. Med J Aust 2011; 194: 16-18.

17 Department of Health (Victoria). Victorian data linkages. http://www.health.vic.gov.au/vdl (accessed Feb 2013).

18 National Centre for Classification in Health. International statistical classification of diseases and related health problems. 10th revision, Australian modification (ICD-10-AM). Sydney: $\mathrm{NCCH}$, 2008. http://meteor.aihw.gov.au/ content/index.phtml/itemld/360927 (accessed Feb 2013).

19 Clothier H, Lee K, Crawford N, et al. Background rates of conditions that may present as potential adverse events following HINI vaccination in Australia [abstract]. 28th Annual Meeting of the European Society of Paediatric Infectious Diseases; 2010 May 4-8; Nice, France. http:// meetings.espid.org/espid2010/abstracts/pdf/ 376.pdf (accessed Mar 2013).

20 Australian Bureau of Statistics. State and regional Indicators, Victoria, Jun 2010. Canberra: ABS, 2010. (ABS Cat. No. 1367.2.) http:// www.abs.gov.au/AUSSTATS/abs@.nsf/ DetailsPage/1367.2Jun+2010 (accessed Feb 2013).

21 Gee J, Naleway A, Shui I, et al. Monitoring the safety of quadrivalent human papillomavirus vaccine: findings from the Vaccine Safety Datalink. Vaccine 2011; 29: 8279-8284.

22 Agorastos T, Chatzigeorgiou K, Brotherton JM, Garland SM. Safety of human papillomavirus (HPV) vaccines: a review of the international experience so far. Vaccine 2009; 27: 7270-7281.

23 Slade BA, Gee J, Broder KR, Vellozzi C. Comment on the contribution by Souayah et al., "GuillainBarré syndrome after Gardasil vaccination: data from Vaccine Adverse Event Reporting System 2006-2009" [letter]. Vaccine 2011; 29: 865-866.

24 Poulos LM, Waters AM, Correll PK, et al. Trends in hospitalizations for anaphylaxis, angioedema, and urticaria in Australia, 1993-1994 to 20042005. J Allergy Clin Immunol 2007; 120: 878-884.

25 Mulla ZD, Lin RY, Simon MR. Perspectives on anaphylaxis epidemiology in the United States with new data and analyses. Curr Allergy Asthma Rep 2011; 11: 37-44.

26 Black S, Eskola J, Siegrist CA, et al. Importance of background rates of disease in assessment of vaccine safety during mass immunisation with pandemic HIN1 influenza vaccines. Lancet 2009; 374: $2115-2122$.

27 Brotherton JM, Deeks SL, Campbell-Lloyd S, et al. Interim estimates of human papillomavirus vaccination coverage in the school-based program in Australia. Commun Dis Intell Q Rep 2008; 32: 457-461.

28 Watson M, Shaw D, Molchanoff L, Mclnnes C. Challenges, lessons learned and results following the implementation of a human papilloma virus school vaccination program in South Australia. Aust N Z J Public Health 2009; 33: 365-370.

29 Farrington $C P$, Whitaker $\mathrm{HJ}$, Hocine MN. Case series analysis for censored, perturbed, or curtailed post-event exposures. Biostatistics 2009; 10: 3-16.

30 Svanström H. Callréus T. Hviid A. Temporal data mining for adverse events following immunization in nationwide Danish healthcare databases. Drug Saf 2010; 33: 1015-1025.

31 Yih WK, Kulldorff M, Fireman BH, et al. Active surveillance for adverse events: the experience of the Vaccine Safety Datalink project. Pediatrics 2011; 127 Suppl 1: S54-S64.

32 Gold MS, Effler P, Kelly H, et al. Febrile convulsions after 2010 seasonal trivalent influenza vaccine: implications for vaccine safety surveillance in Australia. Med J Aust 2010; 193: 492-493. 